\title{
Concurrent Validity of a Commercial Wireless Trunk Triaxial Accelerometer System for Gait Analysis
}

\author{
Roel De Ridder, Julien Lebleu, Tine Willems, Cedric De Blaiser, \\ Christine Detrembleur, and Philip Roosen
}

\begin{abstract}
Context: Wearable sensor devices have notable advantages, such as cost-effectiveness, easy to use, and real-time feedback. Wirelessness ensures full-body motion, which is required during movement in a challenging environment such as during sports. Research on the reliability and validity of commercially available systems, however, is indispensable. Objective: To confirm the test-retest reliability and concurrent validity of a commercially available body-worn sensor-BTS G-WALK ${ }^{\circledR}$ sensor systemfor spatiotemporal gait parameters with the GAITRite ${ }^{\circledR}$ walkway system as golden standard. Design: Reliability and concurrent validity study. Setting: Laboratory setting. Participants: Thirty healthy subjects. Main Outcome Measures: Spatiotemporal parameters: speed, cadence, stride length, stride duration, stance duration, swing duration, double support, and single support. Results: In terms of test-retest reliability of the BTS G-WALK ${ }^{\circledR}$ sensor system, intraclass correlation coefficient values for both the spatial and temporal parameters were excellent between consecutive measurements on the same day with intraclass correlation coefficient values ranging from .85 to .99 . In terms of validity, intraclass correlation coefficient values between measurement systems showed excellent levels of agreement for speed, cadence, stride length, and stride duration (range $=.88-.97$ ), and showed poor to moderate levels of agreement (range $=.12-.47$ ) for single/double support and swing/stance duration. Bland-Altman plots showed overall percentage bias values equal to or smaller than $3 \%$ with limits of agreement $\leq 15 \%$ (speed, cadence, stride length, stride duration, swing duration, and stance duration). Only for single and double support, the limits of agreement were higher with, respectively, $-15.4 \%$ to $19.5 \%$ and $-48.0 \%$ to $51.4 \%$. Conclusion: The BTS G-WALK ${ }^{\circledR}$ sensor system is reliable for all measured spatiotemporal parameters. In terms of validity, excellent concurrent validity was shown for speed, cadence, stride length, and stride duration. Cautious interpretation is necessary for temporal parameters based on final foot contact (stance, swing, and single/ double support time).
\end{abstract}

Keywords: reliability, wearable, spatiotemporal parameters, walking

Wearable sensor devices have notable advantages, such as cost-effectiveness, easy to use, and real-time feedback. Wirelessness ensures full-body motion, which is required during movement in a challenging environment such as during sports. These technology systems allow clinicians to benefit from data gathered during the performance of everyday activities or sports and data recorded under controlled conditions in clinical settings. ${ }^{1}$ For gait analysis, such wearables are potentially useful for assessing abnormal gait and evaluating the effectiveness of rehabilitation approaches and therapeutic interventions.

It has been demonstrated that spatiotemporal gait parameters can be determined during overground walking using only one triaxial trunk accelerometer. ${ }^{2}$ However, to overcome some associated critical issues as the need for gravity compensation and the presence of drift error in the position data, data fusion of linear acceleration (accelerometer) and angular velocity (gyroscope) combined in an inertial measurement unit (IMU) permits compensation. ${ }^{3}$ Notwithstanding the closer the IMU is positioned to the point of contact (eg, on the shank) the better gait events can be correctly detected, ${ }^{4}$ lower trunk acceleration patterns have been

De Ridder, Willems, De Blaiser, and Roosen are with the Department of Rehabilitation Sciences and Physiotherapy, Faculty of Medicine and Health Sciences, Ghent University, Ghent, Belgium. Lebleu and Detrembleur are with Neuro Musculo Skeletal Lab (NMSK), Secteur des Sciences de la Santé, Institut de Recherche Expérimentale et Clinique, Université catholique de Louvain, Brussels, Belgium. De Ridder (Roel.DeRidder@ugent.be) is corresponding author. consistently associated with initial and final foot contact. $^{5}$ Recently, industry transfers technology development made in research ${ }^{6}$ to implement it in clinical routine. For example, the G-WALK is a user-friendly device, without postprocessing handling needed to obtain the gait parameters. This aspect is really important to facilitate technology adoption. ${ }^{7}$ However, before using them for clinical interpretation, we need to define their reliability and validity. The latter can be done by comparing the spatiotemporal parameters of gait obtained from the IMU to a golden standard, such as the GAITRite ${ }^{\circledR}$ system with proven reliability and validity of spatiotemporal gait parameters. ${ }^{8}$

The purpose of this study was to confirm the test-retest reliability and concurrent validity of a commercially available body-worn sensor-BTS G-WALK ${ }^{\circledR}$ sensor system-for spatiotemporal gait parameters in a healthy population with the GAITRite $^{\circledR}$ walkway system as golden standard.

\section{Methods}

\section{Subjects}

Thirty healthy subjects (15 males and 15 females; mean age $=$ $37.8 \mathrm{y}$ and range $=20-56$ ) volunteered to participate in this study. The average weight and height of the subjects are 73.8 (16.84) kg and $173.6(9.42) \mathrm{cm}$, respectively. Subjects had to be healthy and were excluded if they reported to have any musculoskeletal, neurological, or systemic pathology potentially affecting their gait. All participants were informed about study procedures prior 
to signing an institutionally approved informed consent. This study was approved by the ethical committee of the Ghent University Hospital ( $\mathrm{n}^{\circ}$ : BE670201526917).

\section{Experimental Approach and Materials}

The BTS G-WALK ${ }^{\circledR}$ (BTS Bioengineering S.p.A., Garbagnate Milanese, Italy) inertial sensor was placed on the lower back with the center of the device at the fifth lumbar vertebrae (L5; Figure 1). The inertial platform is equipped with 4 sensor fusion technology and consists of a triaxial accelerometer (16 bit/axes; $8 g$ ), a triaxial magnetometer (13 bit; $1200 \mu \mathrm{T})$, a triaxial gyroscope (16 bit/axes; $250 \%$ s), and a global positioning system receiver. The BTS G-WALK ${ }^{\circledR}$ sensor system determines spatiotemporal parameters as well as pelvic rotations during gait and other movements realtime through a Bluetooth ${ }^{\circledR} 3.0$ connection $\left(\mathrm{G}-\right.$ Studio ${ }^{\circledR}$ software). For the current research question, only spatiotemporal parameters were used for further analysis.

The GAITRite $^{\circledR}$ (CIR Systems Inc, Havertown, PA) walkway system used for this study measured $7.03 \mathrm{~m} \times 0.89 \mathrm{~m}$ with an active sensor area of $6.13 \mathrm{~m} \times 0.56 \mathrm{~m}$. The sensors are triggered when mechanical pressure is applied at a used sampling rate of $100 \mathrm{~Hz}$. Data are collected by on-board processors for a connected computer to receive through a serial port (GAITRite ${ }^{\circledR}$ software).

\section{Procedures}

Each subject performed 5 walking trials on the GAITRite ${ }^{\circledR}$ sensor system. All subjects wore their own comfortable and nonrestricting clothing. Every individual was instructed to walk toward the end of the walkway mat at their own everyday pace. During each trial, both the GAITRite ${ }^{\circledR}$ and the BTS G-WALK ${ }^{\circledR}$ sensor systems (G-Studio ${ }^{\circledR}$ software) recorded equivalent parameters to be analyzed

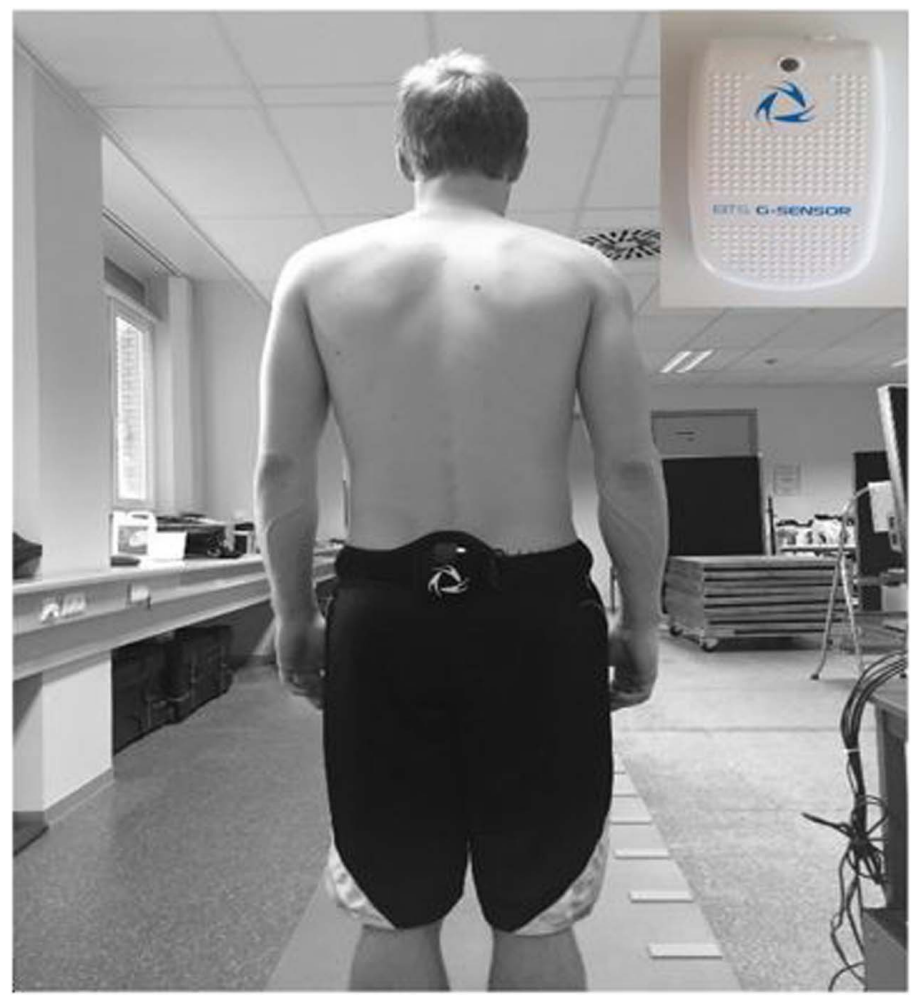

Figure 1 - BTS G-WALK ${ }^{\circledR}$ sensor system. and compared. The parameters that were registered by both systems were speed (meter per second), cadence (steps per minute), stride length (meters), stride duration (seconds), stance duration (percentage of gait cycle [\%GC]), swing duration (\%GC), double support (\% $\mathrm{GC})$, and single support (\%GC).

\section{Data Analysis and Statistics}

Based on comparison with the GAITRite ${ }^{\circledR}$ sensor system, the following methods were used to determine the reliability and validity of the BTS G-WALK ${ }^{\circledR}$ sensor system. For unilateral (left and right) outcome measures, only the data from the right side were included for analysis. Normality of the data was verified using the Shapiro-Wilk test. The reliability of the BTS G-WALK ${ }^{\circledR}$ sensor system outcome measures across the 5 walking trials were examined using intraclass correlation coefficients (ICCs; 2-way mixed, absolute agreement). For establishing validity, means and SDs were calculated for each parameter for both sensor systems over the 5 walking trials. Then, ICCs between the 2 methods were calculated. A paired sample $t$ test was performed to determine systematic differences between the 2 systems. Subsequently, Bland-Altman plots with $95 \%$ limits of agreement (LoA; mean difference $\pm 1.96 \mathrm{SD}$ ) were generated to visualize the degree of agreement between the BTS G-WALK ${ }^{\circledR}$ and the GAITRite ${ }^{\circledR}$ sensor system measurements. Based on the Bland-Altman plots, the percentage bias was calculated with the GAITRite system as reference standard ([mean G-WALK - mean GAITRite]/mean GAITRite $\times 100$ ). Statistical significance for all tests was determined at the $5 \%$ level.

\section{Results}

\section{Intertrial Reliability}

Reliability of all spatiotemporal gait variables recorded by means of the BTS G-WALK ${ }^{\circledR}$ sensor system across the 5 trials is presented in Table 1. Overall, excellent intertrial reliability (ICC values between .84 and .99) is shown.

\section{Concurrent Validity}

Table 1 also displays comparative data for both gait analysis systems. ICC values showed excellent levels of agreement for speed, cadence, stride length, and stride duration (range $=.88-.97$ ), and showed poor to moderate levels of agreement (range $=.12-.47$ ) for the relative temporal parameters that divide the gait cycle in phases (single/double support and swing/stance duration). Paired $t$ tests revealed that there were no significant systematic differences for speed, single and double support $(P>.05)$. Comparison for cadence $(P<.001)$, stride length $(P=.03)$, and stride $(P=.001) /$ swing $(P=.01) /$ stance $(P=.01)$ duration did show a significant difference between devices (Table 1). However, Bland-Altman plots showed overall percentage bias values equal to or smaller than $3 \%$ with LoA $\leq 15 \%$ (speed, cadence, stride length, stride duration, and swing duration; Table 1; Figure 2). Only for single and double support, the LoA were higher with, respectively, $-15.4 \%$ to $19.5 \%$ and $-48.0 \%$ to $51.4 \%$ (Table 1; Figure 2).

\section{Discussion}

In terms of test-retest reliability of the BTS G-WALK ${ }^{\circledR}$ sensor system, ICC values for both the spatial and temporal parameters are 
Table 1 Reliability of G-WALK and Validity Outcome Measures Between G-WALK and GAITRite Measuring Systems

\begin{tabular}{|c|c|c|c|c|c|c|c|c|}
\hline & \multirow[b]{2}{*}{$\begin{array}{l}\text { G-WALK, } \\
\text { Mean (SD) }\end{array}$} & \multirow[b]{2}{*}{$\begin{array}{l}\text { GAITRite, } \\
\text { Mean (SD) }\end{array}$} & \multirow[b]{2}{*}{$\begin{array}{c}\text { G-WALK, } \\
\text { ICC (95\% CI) }\end{array}$} & \multicolumn{2}{|c|}{ Paired sample $t$ test } & \multirow[b]{2}{*}{ Bias, \% } & \multirow[b]{2}{*}{ LoA, \% } & \multirow{2}{*}{$\begin{array}{l}\text { G-WALK vs } \\
\text { GAITRite, } \\
\text { ICC (95\% CI) }\end{array}$} \\
\hline & & & & $\begin{array}{l}\text { Mean difference } \\
(95 \% \mathrm{Cl})\end{array}$ & $P$ value & & & \\
\hline Speed, $\mathrm{m} / \mathrm{s}$ & $1.42(0.21)$ & $1.44(0.17)$ & $.99(.98$ to .99$)$ & $-0.02(-0.06$ to 0.2$)$ & .28 & -1.6 & -15.1 to 11.8 & $.92(.83$ to .96$)$ \\
\hline Cadence, steps/min & $115.3(1.2)$ & $113.0(6.6)$ & $.98(.97$ to .99$)$ & 2.3 (2.0 to 2.7$)$ & $<.001$ & 2.1 & 0.4 to 3.8 & $.96(.02$ to .99$)$ \\
\hline Stride length, m & $1.49(0.19)$ & $1.53(0.14)$ & .99 (.98 to .99$)$ & $-0.04(-0.08$ to -0.004$)$ & .03 & -2.9 & -15.7 to 9.8 & $.88(.73$ to .94$)$ \\
\hline Stride duration, $\mathrm{s}$ & $1.05(0.06)$ & $1.06(0.06)$ & $.84(.73$ to .92$)$ & $-0.02(-0.03$ to -0.004$)$ & .01 & -1.3 & -6.7 to 4.1 & .93 (.83 to .97$)$ \\
\hline Single support, \%GC & $38.7(3.2)$ & $37.9(1.3)$ & $.91(.85$ to .95$)$ & $0.7(-0.5$ to 2.0$)$ & .23 & 2.0 & -15.4 to 19.5 & $.18(-.70$ to .60$)$ \\
\hline Double support, \%GC & $11.0(2.5)$ & $11.0(1.5)$ & $.89(.80$ to .94$)$ & $0.02(-1.0$ to 1.1$)$ & .97 & 1.7 & -48.0 to 51.4 & $.12(-.92$ to .59$)$ \\
\hline Swing duration, \%GC & $39.3(2.3)$ & $38.1(1.3)$ & $.85(.75$ to .92$)$ & $1.1(0.3$ to 1.9$)$ & .01 & 3.0 & -8.5 to 14.4 & $.47(-.04$ to .74$)$ \\
\hline Stance duration, $\% \mathrm{GC}$ & $60.8(2.3)$ & $61.8(1.3)$ & $.85(.75$ to .92$)$ & $-1.1(-1.9$ to -0.3$)$ & .01 & -1.8 & -8.6 to 5.0 & $.47(-.36$ to .74$)$ \\
\hline
\end{tabular}

Abbreviations: CI, confidence interval; GC, gait cycle; ICC, intraclass correlation coefficient; LoA, limits of agreement.
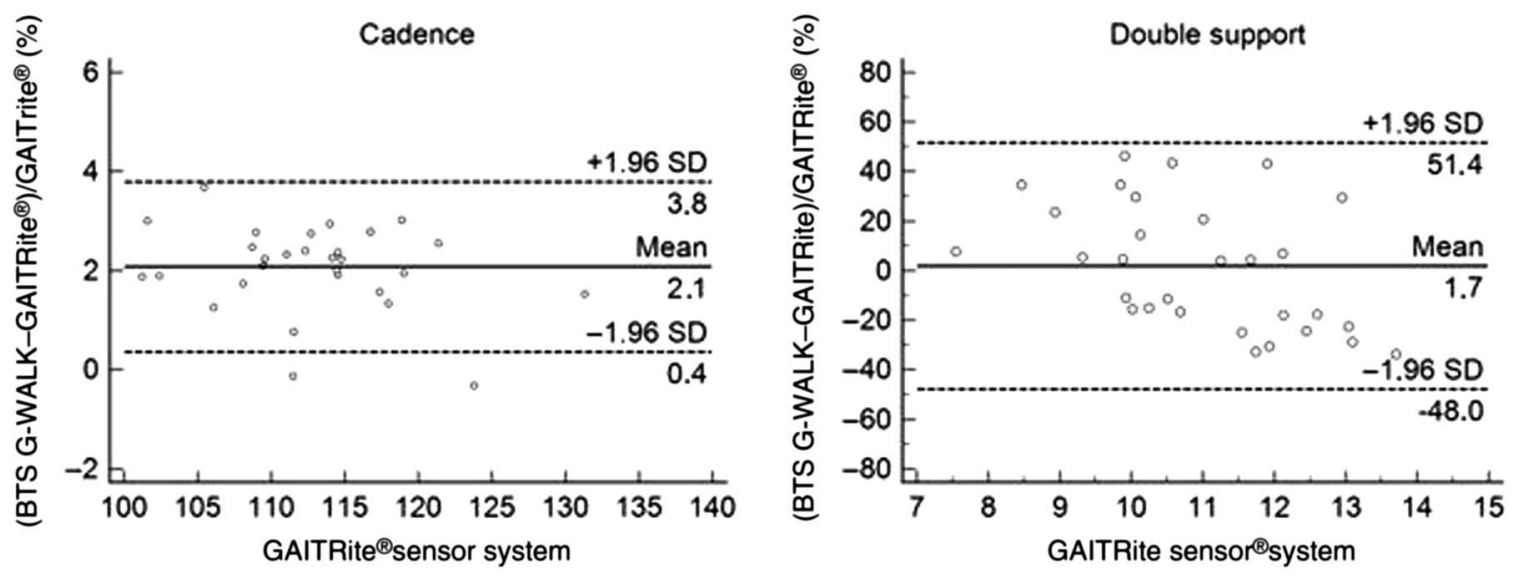

Figure 2 - Bland-Altman plots (\%) with 95\% limits of agreement for cadence and double support.

excellent between consecutive measurements on the same day with ICC values ranging from .85 to .99 . This signifies that the BTS G-WALK ${ }^{\circledR}$ sensor system is reliable for all measured parameters.

Concerning the validity, the results require some careful considerations. First of all, the paired sample $t$ test did show significant differences between the 2 measurement devices for cadence, stride length, and stride/swing/stance duration; with no differences for speed, single and double support. However, when taken the percentage bias into consideration, the overall percentage bias was equal to or lower than $3 \%$, which is within clinically acceptable limits. Speed, cadence, stride length, and stride duration showed excellent ICC values between test devices with LoA for percentage bias $\leq 15 \%$. For swing and stance duration termination, ICC values between devices were moderate to good, also with LoA for percentage bias $\leq 15 \%$. Poor ICC values with LoA for percentage bias $\geq 15 \%$, however, were observed for single and double support. This corresponds with Trojaniello et $\mathrm{al}^{9}$ who evaluated several methods of single IMU's mounted on the lower trunk and showed an acceptable accuracy, sensitivity, and robustness for temporal parameters based on the identification of the initial foot contact, that is, step and stance duration. The accuracy was lower for parameters based on final foot contact (stance, swing, and double support time) as larger errors in event determination of final foot contact were observed. To improve validity, future research should focus on ameliorating IMU algorithms for identifying this final foot contact.

Limitations of the current study are that we are unaware of the precise algorithm used by this commercial IMU, which prohibits us from presenting a potential underlying explanation for the reported results. Furthermore, we measured spatiotemporal parameters at a rather homogenous speed in our sample and speed might have an impact on accuracy. ${ }^{10}$ Spatiotemporal parameters were registered only over a short distance in a controlled setting, and we only included healthy subjects. Reliability and validity (specific parameters) results suggest that the BTS G-WALK ${ }^{\circledR}$ sensor system might be used in community and athletic settings or clinical studies evaluating treatment effects; however, research in these specific populations is warranted.

In conclusion, the BTS G-WALK ${ }^{\circledR}$ sensor system is a reliable IMU for all measured spatiotemporal parameters. In terms of validity, cautious interpretation is necessary for temporal parameters based on final foot contact (stance, swing, and single/double support time). Excellent concurrent validity was shown for speed, cadence, stride length, and stride duration. 


\section{Acknowledgments}

The authors would like to thank Maarten Van Gorp and Christophe Pieters for their help in data collection. The authors have no conflicts of interest to disclose.

\section{References}

1. Bonato P. Advances in wearable technology and applications in physical medicine and rehabilitation. J Neuroeng Rehabil. 2005; 2(1):2. PubMed ID: 15733322 doi:10.1186/1743-0003-2-2

2. Zijlstra W. Assessment of spatio-temporal parameters during unconstrained walking. Eur J Appl Physiol. 2004;92(1-2):39-44. PubMed ID: 14985994 doi:10.1007/s00421-004-1041-5

3. Taborri J, Palermo E, Rossi S, Cappa P. Gait partitioning methods: a systematic review. Sensors. 2016;16(1):E66. doi:10.3390/s16010066

4. Alvarez JC, Alvarez D, Lopez A, Gonzalez RC. Pedestrian navigation based on a waist-worn inertial sensor. Sensors. 2012;12(8):1053610549. doi:10.3390/s120810536

5. Mansfield A, Lyons GM. The use of accelerometry to detect heel contact events for use as a sensor in FES assisted walking. Med Eng Phys. 2003;25(10):879-885. PubMed ID: 14630475 doi:10.1016/ S1350-4533(03)00116-4
6. Gonzalez RC, Lopez AM, Rodriguez-Uria J, Alvarez D, Alvarez JC. Real-time gait event detection for normal subjects from lower trunk accelerations. Gait Posture. 2010;31(3):322-325. PubMed ID: 20034797 doi:10.1016/j.gaitpost.2009.11.014

7. Papi E, Murtagh GM, McGregor AH. Wearable technologies in osteoarthritis: a qualitative study of clinicians' preferences. BMJ Open. 2016;6(1):e009544. PubMed ID: 26810998 doi:10.1136/ bmjopen-2015-009544

8. Webster KE, Wittwer JE, Feller JA. Validity of the GAITRite walkway system for the measurement of averaged and individual step parameters of gait. Gait Posture. 2005;22(4):317-321. PubMed ID: 16274913 doi:10.1016/j.gaitpost.2004.10.005

9. Trojaniello D, Cereatti A, Pelosin E, et al. Estimation of step-by-step spatio-temporal parameters of normal and impaired gait using shankmounted magneto-inertial sensors: application to elderly, hemiparetic, parkinsonian and choreic gait. J Neuroeng Rehabil. 2014;11:152. PubMed ID: 25388296 doi:10.1186/1743-0003-11-152

10. Greene BR, Foran TG, McGrath D, Doheny EP, Burns A, Caulfield B. A comparison of algorithms for body-worn sensor-based spatiotemporal gait parameters to the GAITRite electronic walkway. J Appl Biomech. 2012;28(3):349-355. PubMed ID: 22087019 doi:10.1123/ jab.28.3.349 